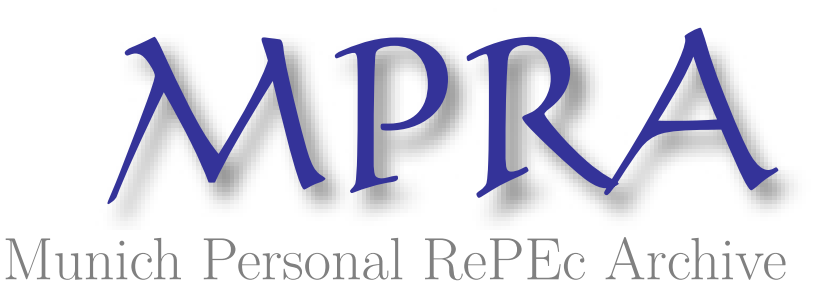

\title{
Contracting under Adverse Selection: Certifiable vs. Uncertifiable Information
}

Schmitz, Patrick W.

University of Cologne

2021

Online at https://mpra.ub.uni-muenchen.de/105106/

MPRA Paper No. 105106, posted 05 Jan 2021 22:21 UTC 


\title{
Contracting under Adverse Selection: Certifiable vs. Uncertifiable Information
}

\author{
Patrick W. Schmitz* \\ University of Cologne, Germany, and CEPR, London, UK
}

\begin{abstract}
The analysis of adverse selection problems in seller-buyer relationships has typically been based on the assumption that private information is uncertifiable, while in practice it may well be certifiable. If a buyer has certifiable private information, he can conceal evidence, but he cannot claim to have information for which he has no evidence, so he has fewer possibilities to misrepresent his information. Nevertheless, we find that the expected total surplus can be strictly smaller in the case of certifiable information than in the case of uncertifiable information. This finding holds when the buyer may have private information with some exogenous probability as well as in the case of opportunistic information gathering, where the buyer can privately decide whether or not to acquire information for strategic reasons.
\end{abstract}

Keywords: Contracting; Asymmetric information; Adverse selection; Screening; Information gathering JEL Classification: D86; D82; D23; C73; L24

* University of Cologne, Department of Economics, Albertus-Magnus-Platz, 50923 Cologne, Germany. E-mail address: patrick.schmitz@uni-koeln.de. I have benefitted from very helpful discussions with Susanne Goldlücke, Franziska Heinicke, Stephanie Rosenkranz, and Urs Schweizer.

This is the working paper version of the following publication:

Schmitz, P.W. (2021). Contracting under Adverse Selection: Certifiable vs. Uncertifiable Information. Journal of Economic Behavior and Organization, Vol. 182, 100-112. 


\section{Introduction}

In practice, relationships between buyers and sellers are often plagued by information asymmetries. For example, a manufacturer may have a more accurate demand forecast for a product than his supplier. In M\&A settings, the management of the target company may have private information about its intangible assets. However, most contract-theoretic studies of seller-buyer contracting problems assume that private information is uncertifiable (i.e., "soft"), so a privately informed buyer can make arbitrary claims about a subjective information parameter. Uncertifiable private information is a plausible assumption in some economic applications, e.g. when a manager faces a worker who has private information about the disutility caused by exerting effort or when a retailer faces consumers who have private information about their tastes.

Yet, in particular in business-to-business relationships it may well be the case that private information can be authenticated in a more objective way. For example, a manufacturer might have verifiable evidence about his production technology and about market studies that are indicative of the profit that he will be able to make with a supplier's intermediate product. The management of a target company might have verifiable evidence about the value of its intangible assets. In the case of certifiable (i.e., "hard") information, a privately informed buyer can always claim to be uninformed (i.e., he can conceal evidence), but he cannot claim to have information for which he has no evidence. ${ }^{1}$

In the first part of the paper, we study a simple adverse selection (i.e., screening) problem in which a seller offers a contract to a buyer who may have private information with some exogenous probability. ${ }^{2}$ At first glance, one might guess

\footnotetext{
${ }^{1}$ Our usage of the terms "uncertifiable" and "certifiable" information follows Bolton and Dewatripont's (2005, chapter 5) leading textbook on the theory of contracting.

${ }^{2}$ Following Hart and Holmström (1987, p. 76), situations in which the agent may have precontractual private information are usually categorized under the heading of "adverse selection" models. In contrast, in "moral hazard" models there is symmetric information at the time of contracting. For example, in a moral hazard problem a worker may exert unobservable effort after the contract has been signed. Moral hazard models often focus on risk-averse agents and the trade-off between incentives and insurance, while in adverse selection models the focus is usually on risk-neutral decision makers (see Laffont and Martimort, 2002; Bolton and Dewatripont, 2005).
} 
that the total surplus generated by the two parties will be larger if the buyer's information is certifiable, since in this case the buyer has fewer possibilities to lie than in the case of uncertifiable information. Yet, we will show that there are situations in which the expected total surplus is strictly smaller when the buyer's information is certifiable.

The intuition behind this finding is as follows. Suppose that the buyer is informed with a relatively high probability, but it is unlikely that his expected revenue from trade is large. In the case of uncertifiable information, trade always takes place, because the seller does not want to risk losing opportunities to trade by offering a contract where the buyer has to make a large payment. Yet, in the case of certifiable information, the seller has more instruments at her disposal, since she can ask the buyer to present evidence. Thus, the seller offers a contract where the buyer has to make a large payment, but the payment is reduced if the buyer proves that his expected revenue is small. However, in this way an uninformed buyer is excluded from trading. In other words, in this case the seller does not use her additional instruments to create a larger pie, but to decrease the buyer's fraction of the pie, thereby reducing its size.

In the second part of the paper, the buyer can endogenously decide whether or not to acquire private information. In our setup, the buyer may gather information for opportunistic reasons; i.e., in order to increase his own profit at the expense of the total gains from trade. For reasons similar to those in the case of exogenous information, it again turns out that the expected total surplus can be strictly smaller in the case of certifiable information than in the case of uncertifiable information. Taken together, we conclude that certifiable private information and strategic information acquisition may lead to serious frictions in seller-buyer contracting, the implications of which deserve to be more thoroughly investigated in future research.

Organization of the paper. The remainder of the paper is organized as follows. In Section 2, we briefly discuss related literature. In Section 3, the basic model is introduced. In Section 4, we study the case of an exogenously given information structure. The information structure is endogenized in Section 5. Finally, con- 
cluding remarks follow in Section 6. Some technical details have been relegated to the Appendix.

\section{Related literature}

To the best of my knowledge, the present paper is the first one that compares the inefficiencies caused by certifiable and uncertifiable information in screening problems with exogenous and endogenous information structures. In screening problems, an uninformed principal offers a contract to a privately informed agent. Most papers in the voluminous literature on screening focus on situations in which the agent is known to have uncertifiable information. The classic papers are Baron and Myerson (1982), who study the regulation of a monopolistic firm that has private information about its costs, and Maskin and Riley (1984), who study monopolistic price discrimination when customers have private information about their valuations. A key finding of these papers is that trade is at its first-best level (i.e., the level that maximizes the total surplus) for the best possible type (the so-called "no distortion at the top" result), while otherwise there is a downward distortion of the trade level. Lewis and Sappington (1993) have extended the analysis of screening problems with uncertifiable information to the case in which the agent may be uninformed with an exogenously given probability. Allowing for the possibility that an agent may be ignorant can lead to even more severe distortions away form the first-best solution.

With regard to certifiable information, following Grossman and Hart (1980) there is a literature focused on signaling problems, where a privately informed principal offers a contract to uninformed agents. In the context of takeover bids, Grossman and Hart (1980) discuss the effects of requiring disclosure of certifiable information as opposed to simply outlawing the making of false statements. A key finding is that if it is commonly known that the principal is informed with certainty, an unravelling result holds according to which the principal fully discloses all of his private information. ${ }^{3}$

\footnotetext{
${ }^{3}$ Cf. Milgrom (1981) and Okuno-Fujiwara et al. (1990). Bolton and Dewatripont (2005,
} 
In a screening model where buyers may have certifiable information, Schmitz (2007) shows that more competition (i.e., increasing the number of buyers) can reduce the expected total surplus.

Moreover, certifiable information has also been studied in the context of collusion in a principal/supervisor/agent setting by Tirole (1986). ${ }^{4}$ In his model, the supervisor may learn evidence about the agent's type. The supervisor can conceal the evidence or reveal it to the principal, but the evidence cannot be forged. In contrast to the present setting, in Tirole (1986) the agent always learns the state of the world. Moreover, in Tirole (1986) the agent has to be induced to exert unobservable effort (i.e., there is a moral hazard problem), while in our setting the trade level is verifiable.

Crémer and Khalil (1992) have initiated a still relatively small literature on screening problems with endogenous information, where the agent can privately decide whether or not to incur costs in order to gather information. They consider a firm that produces spare parts under contract for another firm. The latter firm suddenly asks for a modification, but initially the first firm does not know how costly the modification will be. Crémer and Khalil (1992) have pointed out that while most papers in the adverse selection literature assume that asymmetric information is exogenously given, the fundamental asymmetry may actually lie in the ability to gather information. So far, in this literature it is assumed that information is uncertifiable. ${ }^{5}$

Taken together, the present paper contributes to the contract-theoretic literature on screening problems by comparing the frictions caused by uncertifiable and certifiable information, allowing for the possibility that the buyer may acquire

chapter 5) provide a comprehensive review of this literature.

${ }^{4}$ See also Laffont and Martimort (1999) for a related study of collusion in a three-tier hierarchy with hard information.

${ }^{5}$ While Crémer and Khalil (1992), Crémer et al. (1998a), and Hoppe and Schmitz (2013a) study opportunistic information acquisition that serves strategic purposes only, other papers such as Lewis and Sappington (1997), Crémer et al. (1998b), Iossa and Martimort (2015), Su (2017a), and Ye and Li (2018) study productive information gathering. Cf. Hoppe and Schmitz (2010), who point out that information gathering can be a pure rent-seeking activity also in settings different from the one initially considered by Crémer and Khalil (1992), in which information becomes costlessly revealed at a later point in time. 
private information for strategic reasons.

\section{The model}

Consider two risk-neutral parties, a seller (she) and a buyer (he), who can trade an intermediate product. For example, the seller may be a biotech firm that has invented a novel technology. The buyer may be a pharmaceutical firm that can benefit from the innovation, since it might be useful to produce existing products at lower costs or to come up with new products. ${ }^{6}$ However, it is uncertain how valuable the innovation will actually turn out to be. For instance, there may be demand uncertainties regarding the buyer's final product. Let $R \geq 0$ denote the buyer's uncertain future revenue from acquiring the seller's innovation, where $R$ is a random variable with a commonly known distribution. We suppose that $R$ is not contractible, since it may realize only in the distant future and since the additional profits the buyer makes based on the seller's input cannot be cleanly disentangled from the buyer's other business activities.

The sequence of events is as follows. At date 1, the seller offers to sell her intermediate product to the buyer in exchange for a transfer payment $t$. At date 2 , the buyer decides whether or not to accept the seller's offer. If the buyer rejects, both parties' payoffs are normalized to zero. If the buyer accepts, his profit will be $q R-t$ and the seller's profit is $t$, where $q \in[0,1]$ can be interpreted as quantity of a divisible good or as probability of trade of an indivisible good. The quantity $q$ is chosen by the seller in line with the agreed-upon contract, since it is a verifiable decision that can be enforced by the court.

At date 0 , there is a random draw $\omega \in\{H, L\}$ by nature. ${ }^{7}$ In the good state of the world, which occurs with probability $\rho \in(0,1)$, the buyer's expected revenue

\footnotetext{
${ }^{6}$ In the present paper we do not study ownership structures, i.e. whether or not vertical integration is optimal. With regard to contract-theoretic studies on the latter question, see Hart (1995) for the case of symmetric information and Schmitz (2006) for the case of (uncertifiable) asymmetric information.

${ }^{7}$ We focus on the two-types case since it allows us to present our novel insights in the simplest way. Indeed, Laffont and Martimort (2002, p. 134) have pointed out "the fact that few new economic insights can be obtained in the continuum case" regarding types in adverse selection models.
} 
is $R_{H}=E[R \mid \omega=H]$. In the bad state of the world, the buyer's expected revenue is only $R_{L}=E[R \mid \omega=L]$, where $0<R_{L}<R_{H}$. At date 0 , the buyer may obtain private information about how valuable the seller's intermediate product might really turn out to be for the buyer's business activities. Specifically, the buyer learns the state of the world $\omega$ with probability $\nu \in[0,1]$, and remains uninformed otherwise. Hence, the buyer privately observes a signal $\sigma \in\{H, L, \emptyset\}$. With probability $\rho \nu$ the signal is $\sigma=H$, in which case the buyer knows that his expected revenue is $R_{H}$. With probability $(1-\rho) \nu$ the signal is $\sigma=L$, so the buyer knows his expected revenue is $R_{L}$. Finally, with probability $1-\nu$ the signal is $\sigma=\emptyset$, which means that the buyer has no information over and above what is common knowledge, so his expected revenue is $E[R]=\rho R_{H}+(1-\rho) R_{L}{ }^{8}$

In what follows, we will compare two scenarios. In scenario $U$, information is uncertifiable. Hence, the buyer can make any claim $\hat{\sigma} \in\{H, L, \emptyset\}$ about the signal $\sigma$, regardless of its actual realization. This is the kind of private information that is most often studied in the existing literature on screening problems.

In scenario $C$, information is certifiable. This means that the buyer can always claim to be uninformed (even when this is not true), but he can claim that he has received the signal $\sigma=L$ or $\sigma=H$ only if this is actually the case. Hence, the buyer's admissible claims in scenario $C$ are $\hat{\sigma}=\emptyset$ if $\sigma=\emptyset, \hat{\sigma} \in\{H, \emptyset\}$ if $\sigma=H$, and $\hat{\sigma} \in\{L, \emptyset\}$ if $\sigma=L$. Observe that in scenario $C$ the buyer has fewer possibilities to be dishonest than in scenario $U$.

\section{Exogenous information}

\subsection{Scenario $U$ : Uncertifiable information}

We first consider scenario $U$, where the buyer is free to make any claim $\hat{\sigma} \in$ $\{H, L, \emptyset\}$ about the signal $\sigma$, since his claim cannot be verified regardless of the

\footnotetext{
${ }^{8}$ Note that for simplicity of the exposition the sellers's costs are assumed to be zero; i.e., she already has produced the intermediate good. The results would not change qualitatively if the seller incurred costs $q C$, where $C<R_{L}$. We thus focus on the case in which the two parties' total profit is maximized by $q=1$ regardless of the realization of $\omega$, so that information acquisition (to be studied in Section 5) can occur for strategic reasons only.
} 
state of the world.

We follow the mechanism design approach and look for the optimal contract given the relevant information structure. In this way, one can characterize the optimal mechanism without imposing any ad hoc restrictions on the class of feasible contracts that do not follow from the informational constraints. In particular, according to the revelation principle, in order to find the seller's maximum expected profit we can without loss of generality confine our attention to direct revelation mechanisms (see Myerson, 1982). In such a mechanism, the buyer is asked about the realization of the signal $\sigma$. The mechanism specifies the trade level $q_{\hat{\sigma}} \in[0,1]$ and the transfer payment $t_{\hat{\sigma}}$ depending on the buyer's claim $\hat{\sigma} \in\{H, L, \emptyset\}$. The trade level and the transfer payment must be designed such that it is in the buyer's self-interest to report the truth. In the present context this means that the seller offers a menu of contracts $\left(q_{H}, t_{H}\right),\left(q_{L}, t_{L}\right),\left(q_{\emptyset}, t_{\emptyset}\right)$, such that a buyer who has observed $\sigma \in\{H, L, \emptyset\}$ will actually pick the contract that was intended for him.

It should be noted that the revelation principle does not say that in practice the parties should use direct revelation mechanisms. Instead, direct revelation mechanisms are just an analytical tool to characterize the seller's maximum expected profit. According to the revelation principle, it is impossible to find a contract that could yield a larger expected profit than the optimal direct revelation mechanism. Once we have derived the maximum expected profits, we can provide simple contracts that will yield the same outcome as the optimal direct revelation mechanisms.

Now let us look for the optimal direct revelation mechanism. The seller's problem is to maximize her expected profit

$$
\nu\left[\rho t_{H}+(1-\rho) t_{L}\right]+(1-\nu) t_{\emptyset}
$$

subject to the relevant constraints. Specifically, if $\sigma=H$, it will be in the buyer's self-interest to truthfully reveal the signal whenever the incentive-compatibility constraints

$$
q_{H} R_{H}-t_{H} \geq q_{L} R_{H}-t_{L} \quad\left(I C_{H L}\right)
$$


and

$$
q_{H} R_{H}-t_{H} \geq q_{\emptyset} R_{H}-t_{\emptyset}
$$

hold. The constraint $\left(I C_{H L}\right)$ ensures that the buyer does not claim that the signal is $L$, while the constraint $\left(I C_{H \emptyset}\right)$ ensures that he does not claim to be uninformed. Similarly, the constraints

$$
\begin{gathered}
q_{L} R_{L}-t_{L} \geq q_{H} R_{L}-t_{H}, \\
q_{L} R_{L}-t_{L} \geq q_{\emptyset} R_{L}-t_{\emptyset}
\end{gathered}
$$

make sure that the buyer will report the signal $\sigma=L$ truthfully. Moreover, the incentive-compatibility constraints with regard to the signal $\sigma=\emptyset$ are

$$
\begin{array}{ll}
q_{\emptyset} E[R]-t_{\emptyset} \geq q_{H} E[R]-t_{H}, & \left(I C_{\emptyset H}\right) \\
q_{\emptyset} E[R]-t_{\emptyset} \geq q_{L} E[R]-t_{L} . & \left(I C_{\emptyset L}\right)
\end{array}
$$

Finally, in order to ensure that it is individually rational for the buyer to accept the seller's offer, the participation constraints

$$
\begin{gathered}
q_{H} R_{H}-t_{H} \geq 0, \\
q_{L} R_{L}-t_{L} \geq 0, \\
q_{\emptyset} E[R]-t_{\emptyset} \geq 0
\end{gathered}
$$

must be satisfied.

Observe that the constraints $\left(I C_{H \emptyset}\right)$ and $\left(I C_{\emptyset H}\right)$ imply $q_{H}\left(R_{H}-E[R]\right) \geq$ $q_{\emptyset}\left(R_{H}-E[R]\right)$, so the monotonicity constraint $q_{H} \geq q_{\emptyset}$ must hold. Similarly, the constraints $\left(I C_{\emptyset L}\right)$ and $\left(I C_{L \emptyset}\right)$ imply the monotonicity constraint $q_{\emptyset} \geq q_{L}$.

We now consider a relaxed problem in which we ignore some of the constraints. Specifically, we maximize the seller's expected profit subject to the constraints $\left(I C_{H \emptyset}\right),\left(I C_{\emptyset L}\right),\left(P C_{L}\right)$, and $q_{L} \leq q_{\emptyset} \leq q_{H}$. It will turn out that the solution of the relaxed problem satisfies the ignored constraints, hence it is also the solution 
of the original problem. ${ }^{9}$

In the solution of the relaxed problem the constraint $\left(P C_{L}\right)$ must be binding, because otherwise we could increase the seller's expected profit by slightly increasing $t_{L}$ without violating any of the remaining constraints. Thus, it is optimal for the seller to set

$$
t_{L}=q_{L} R_{L}
$$

Similar arguments show that the constraints $\left(I C_{\emptyset L}\right)$ and $\left(I C_{H \emptyset}\right)$ must be binding. Hence, it is optimal for the seller to set

$$
\begin{gathered}
t_{\emptyset}=q_{\emptyset} E[R]-q_{L}\left(E[R]-R_{L}\right), \\
t_{H}=q_{H} R_{H}-q_{\emptyset}\left(R_{H}-E[R]\right)-q_{L}\left(E[R]-R_{L}\right) .
\end{gathered}
$$

We can thus further simplify the seller's problem by eliminating the transfer payments. The seller chooses $q_{H}, q_{\emptyset}$, and $q_{L}$ in order to maximize her expected profit

$$
\nu \rho R_{H} q_{H}+\left[(1-\nu) E[R]-\nu \rho\left(R_{H}-E[R]\right)\right] q_{\emptyset}+\left[\nu(1-\rho) E[R]-\left(E[R]-R_{L}\right)\right] q_{L}
$$

subject to $0 \leq q_{L} \leq q_{\emptyset} \leq q_{H} \leq 1$. We define three threshold functions.

Definition 1 Let $\hat{\nu}(\rho)=\frac{E[R]}{E[R]+\left(R_{H}-E[R]\right) \rho}, \bar{\nu}(\rho)=\frac{E[R]-R_{L}}{E[R](1-\rho)}$, and $\tilde{\nu}(\rho)=\frac{R_{L}}{\rho R_{H}}$.

As shown in the proof of Lemma 1 below, the sign of the coefficient of $q_{\emptyset}$ (resp., $\left.q_{L}\right)$ in the seller's objective function depends on whether $\nu$ is larger or smaller than the threshold $\hat{\nu}(\rho)$ (resp., $\bar{\nu}(\rho))$. Observe that $\hat{\nu}(\rho) \in(0,1), \bar{\nu}(\rho)>0$, and $\tilde{\nu}(\rho)>0$. It is straightforward to show that there exists a critical value $\hat{\rho} \in(0,1)$, such that $\rho \leq \hat{\rho}$ implies $\bar{\nu}(\rho) \leq \hat{\nu}(\rho) \leq \tilde{\nu}(\rho)$, and $\rho \geq \hat{\rho}$ implies $\tilde{\nu}(\rho) \leq \hat{\nu}(\rho) \leq \bar{\nu}(\rho) \cdot{ }^{10}$ Therefore, the following findings completely characterize the solution to the seller's problem.

Lemma 1 It is optimal for the seller to set $q_{H}=1$ and to set $q_{L}$ and $q_{\emptyset}$ as follows.

\footnotetext{
${ }^{9}$ For details, see Observation 1 in the Appendix.

${ }^{10}$ See Observation 2 in the Appendix.
} 
(i) Suppose $\nu>\hat{\nu}(\rho)$. If $\nu>\tilde{\nu}(\rho)$, then $q_{L}=q_{\emptyset}=0$. If $\nu=\tilde{\nu}(\rho)$, then $0 \leq q_{L}=q_{\emptyset} \leq 1$.

(ii) Suppose $\nu<\bar{\nu}(\rho)$. If $\nu<\hat{\nu}(\rho)$, then $q_{L}=0$, $q_{\emptyset}=1$. If $\nu=\hat{\nu}(\rho)$, then $q_{L}=0$, $0 \leq q_{\emptyset} \leq 1$.

(iii) Suppose $\nu<\tilde{\nu}(\rho)$. If $\nu>\bar{\nu}(\rho)$, then $q_{L}=q_{\emptyset}=1$. If $\nu=\bar{\nu}(\rho)$, then $0 \leq q_{L} \leq 1, q_{\emptyset}=1$.

(iv) If $\nu=\bar{\nu}(\rho)=\tilde{\nu}(\rho)$ and hence $\rho=\hat{\rho}$, then $0 \leq q_{L} \leq 1$ and $q_{L} \leq q_{\emptyset} \leq 1$.

Proof of Lemma 1. First observe that in the objective function the coefficient of $q_{H}$ is positive, hence it is optimal for the seller to set $q_{H}=1$. Next, with regard to the coefficient of $q_{\emptyset}$, the condition $(1-\nu) E[R]-\nu \rho\left(R_{H}-E[R]\right) \geq 0$ is satisfied whenever $\nu \leq \hat{\nu}(\rho)$. Regarding the coefficient of $q_{L}$, observe that $\nu(1-\rho) E[R]-\left(E[R]-R_{L}\right) \geq 0$ whenever $\nu \geq \bar{\nu}(\rho)$.

As a consequence, the following behavior is optimal for the seller. If $v<\hat{\nu}(\rho)$, the seller sets $q_{\emptyset}=1$. If $v=\hat{\nu}(\rho)$, the seller is indifferent between all $q_{\emptyset}$ in the unit interval. If $v>\hat{\nu}(\rho)$, she sets $q_{\emptyset}=q_{L}$. Analogously, if $\nu<\bar{\nu}(\rho)$ the seller sets $q_{L}=0$. If $\nu=\bar{\nu}(\rho)$, she is indifferent between all $q_{L}$ in the unit interval. If $\nu>\bar{\nu}(\rho)$, she sets $q_{L}=q_{\emptyset}$. Moreover, the seller prefers $q_{L}=q_{\emptyset}=1$ to $q_{L}=q_{\emptyset}=0$ whenever $\nu$ is smaller $\tilde{\nu}(\rho)$. Hence, the claims made in the lemma follow immediately. Observe that case (ii) can occur only if $\rho \leq \hat{\rho}$. Notice that the lemma covers all conceivable cases due to Observation 2 which is shown in the Appendix.

Note that the cases in which the seller is indifferent between several contracts will become relevant when we endogenize the information structure (see Section 5). For now we may simply assume as a tie breaking convention that in case of indifference the seller offers the contract that is best for the buyer.

Proposition 1 Consider scenario U (uncertifiable information).

(i) If $\nu>\hat{\nu}(\rho)$ and $\nu>\tilde{\nu}(\rho)$, the seller offers the menu $\left(q_{H}=1, t_{H}=R_{H}\right)$, $\left(q_{L}=0, t_{L}=0\right),\left(q_{\emptyset}=0, t_{\emptyset}=0\right)$. The seller's expected profit is $\nu \rho R_{H}$, the buyer's expected profit is 0 , and hence the expected total surplus is $S_{U}=\nu \rho R_{H}$. 
(ii) If $\nu \leq \hat{\nu}(\rho)$ and $\nu<\bar{\nu}(\rho)$, the seller offers $\left(q_{H}=1, t_{H}=E[R]\right),\left(q_{L}=0\right.$, $\left.t_{L}=0\right),\left(q_{\emptyset}=1, t_{\emptyset}=E[R]\right)$. The seller's expected profit is $(1-\nu) E[R]+\nu \rho E[R]$, the buyer's expected profit is $\nu \rho\left(R_{H}-E[R]\right)$, and the expected total surplus is $S_{U}=(1-\nu) E[R]+\nu \rho R_{H}$.

(iii) If $\bar{\nu}(\rho) \leq \nu \leq \tilde{\nu}(\rho)$, the seller offers $\left(q_{H}=1, t_{H}=R_{L}\right),\left(q_{L}=1, t_{L}=R_{L}\right)$, $\left(q_{\emptyset}=1, t_{\emptyset}=R_{L}\right)$. The seller's expected profit is $R_{L}$, the buyer's expected profit is $E[R]-R_{L}$, and the expected total surplus is $S_{U}=E[R]$.

Intuitively, the seller faces a trade-off between achieving an ex post efficient outcome and reducing the buyer's information rent. In case (i), the buyer is informed with a large probability, and trade will occur only if the buyer knows that we are in the good state of the world. ${ }^{11}$ While the outcome is not always ex post efficient, it has the advantage that the seller does not have to leave a rent to the buyer. In contrast, if the buyer is informed with a very small probability (case ii), trade also takes place if the buyer is uninformed. This implies that when the buyer knows that we are in the good state (which happens with probability $\nu \rho$ ), he must get a rent. Note that an ex post inefficiency occurs if the buyer is informed (which is relatively unlikely in case ii) and we are in the bad state of the world. Finally, when the buyer is informed with an intermediate probability (case iii), trade always takes place, so ex post efficiency is achieved, and the buyer gets the expected rent $E[R]-R_{L}$, which is larger than the expected rent $\nu \rho\left(R_{H}-E[R]\right)$ in case (ii).

As pointed out above, the revelation principle ensures that by characterizing the seller's optimal direct revelation mechanism, we can be sure that there exists no contract whatsoever that could yield a larger expected profit for the seller. Once we know the maximum expected profit, we can look for simple contracts that attain this profit level. In the present case, observe that the seller can attain her maximum expected profit by making a posted-price offer $p$, which the buyer

\footnotetext{
${ }^{11}$ Note that $\nu>\tilde{\nu}(\rho)$ can hold only if $\rho R_{H}>R_{L}$, hence it is more profitable for the seller to trade only in the good state (so she can demand the payment $R_{H}$ ) than to also trade in the bad state (asking for the payment $R_{L}$ only). Moreover, when $\nu>\hat{\nu}(\rho)$ the event that the buyer is uninformed is sufficiently unlikely, such that the seller refrains from trading also in this event (since then she could demand the payment $E[R]$ only).
} 
may either accept or reject. Specifically, if $\nu>\hat{\nu}(\rho)$ and $\nu>\tilde{\nu}(\rho)$ it is optimal for the seller to set $p=R_{H}$. Indeed, only a buyer who observed the signal $\sigma=H$ will accept the offer $p=R_{H}$. Hence, the same allocation will be implemented as in case (i) of Proposition 1. Similarly, in case (ii) it is optimal for the seller to set $p=E[R]$, and in case (iii) it is optimal for her to set $p=R_{L}$. To see this, note that an offer $p=E[R]$ will be accepted by the buyer if he has observed a signal $\sigma \in\{H, \emptyset\}$, while an offer $p=R_{L}$ will always be accepted.

\subsection{Scenario $C$ : Certifiable information}

We now study scenario $C$, where the buyer can no longer claim to have observed $\sigma=L$ or $\sigma=H$ without presenting the underlying evidence. However, it is still the case that the buyer can always claim to be uninformed $(\sigma=\emptyset)$, even if this is not true.

We again do not impose any ad hoc restrictions on the class of feasible contracts. Thus, we consider direct revelation mechanisms that specify the trade level $q_{\hat{\sigma}} \in[0,1]$ and the transfer payment $t_{\hat{\sigma}}$ depending on the buyer's claim $\hat{\sigma} \in\{H, L, \emptyset\} .12$

The seller's problem is thus to find a menu $\left(q_{H}, t_{H}\right),\left(q_{L}, t_{L}\right),\left(q_{\emptyset}, t_{\emptyset}\right)$ that maximizes her expected profit

$$
\nu\left[\rho t_{H}+(1-\rho) t_{L}\right]+(1-\nu) t_{\emptyset}
$$

subject to the relevant constraints. Specifically, if the buyer has observed the signal $\sigma=H$, he has no incentive to conceal this information whenever the incentivecompatibility constraint

$$
q_{H} R_{H}-t_{H} \geq q_{\emptyset} R_{H}-t_{\emptyset}
$$

\footnotetext{
${ }^{12}$ See Green and Laffont (1986) for the revelation principle in the case of partially verifiable information.
} 
is satisfied. Moreover, the constraint

$$
q_{L} R_{L}-t_{L} \geq q_{\emptyset} R_{L}-t_{\emptyset}
$$

ensures that the buyer reveals the truth if $\sigma=L$. In addition, to make sure that it is individually rational for the buyer to accept the seller's offer, the participation constraints

$$
\begin{gathered}
q_{H} R_{H}-t_{H} \geq 0, \\
q_{L} R_{L}-t_{L} \geq 0, \\
q_{\emptyset} E[R]-t_{\emptyset} \geq 0
\end{gathered}
$$

must hold. Observe that there are fewer constraints than in the case of uncertifiable information, so it is clear that in scenario $C$ the seller cannot be worse off than in scenario $U$.

The constraint $\left(P C_{H}\right)$ is redundant, because it already follows from $\left(I C_{H \emptyset}\right)$ and $\left(P C_{\emptyset}\right)$, due to the fact that $R_{H}>E[R]$. The constraint $\left(I C_{H \emptyset}\right)$ must be binding, since otherwise the seller's expected profit could be increased by slightly increasing $t_{H}$, without violating any of the remaining constraints. Similarly, the participation constraint $\left(P C_{\emptyset}\right)$ must be binding. Hence, it is optimal for the seller to set

$$
\begin{gathered}
t_{\emptyset}=q_{\emptyset} E[R], \\
t_{H}=q_{H} R_{H}-q_{\emptyset}\left(R_{H}-E[R]\right) .
\end{gathered}
$$

Note that $\left(P C_{L}\right)$ must be binding, because the right-hand side of constraint $\left(I C_{L \emptyset}\right)$ satisfies $q_{\emptyset} R_{L}-t_{\emptyset} \leq q_{\emptyset} E[R]-t_{\emptyset}=0$. Thus,

$$
t_{L}=q_{L} R_{L}
$$

We can now eliminate the transfer payments from the maximization problem. The seller chooses $q_{H}, q_{\emptyset}$, and $q_{L}$ in order to maximize her expected profit

$$
\nu \rho R_{H} q_{H}+\left[(1-\nu) E[R]-\nu \rho\left(R_{H}-E[R]\right)\right] q_{\emptyset}+\nu(1-\rho) R_{L} q_{L} .
$$


Note that $\nu \rho R_{H}>0$ and $\nu(1-\rho) R_{L}>0$. Furthermore, the coefficient of $q_{\emptyset}$ is positive whenever $(1-\nu) E[R]-\nu \rho\left(R_{H}-E[R]\right) \geq 0$ is satisfied, which is the case whenever $\nu \leq \hat{\nu}(\rho)$. Thus, the following result must hold.

Lemma 2 In the solution to the seller's problem, $q_{H}=q_{L}=1$. Moreover, if $\nu>\hat{\nu}(\rho)$, then $q_{\emptyset}=0$. If $\nu<\hat{\nu}(\rho)$, then $q_{\emptyset}=1$. If $\nu=\hat{\nu}(\rho)$, then $0 \leq q_{\emptyset} \leq 1$.

We have now found the solution to the seller's problem in the case of certifiable information.

Proposition 2 Consider scenario $C$ (certifiable information).

(i) If $\nu>\hat{\nu}(\rho)$, the seller offers the menu $\left(q_{H}=1, t_{H}=R_{H}\right),\left(q_{L}=1, t_{L}=R_{L}\right)$, $\left(q_{\emptyset}=0, t_{\emptyset}=0\right)$. The seller's expected profit is $\nu E[R]$, the buyer's expected profit is 0 , and hence the expected total surplus is $S_{C}=\nu E[R]$.

(ii) If $\nu \leq \hat{\nu}(\rho)$, the seller offers $\left(q_{H}=1, t_{H}=E[R]\right),\left(q_{L}=1, t_{L}=R_{L}\right),\left(q_{\emptyset}=1\right.$, $\left.t_{\emptyset}=E[R]\right)$. The seller's expected profit is $E[R]-\nu \rho\left(R_{H}-E[R]\right)$, the buyer's expected profit is $\nu \rho\left(R_{H}-E[R]\right)$, and the expected total surplus is $S_{C}=E[R]$.

The seller again faces a trade-off between achieving ex post efficiency and reducing the buyer's rent. If the buyer is informed with a large probability (case i), then trade takes place only if the buyer can provide evidence for the prevailing state of the world. In this case, the buyer does not get a rent. In contrast, if the buyer is informed with a small probability (case ii), trade always takes place, so ex post efficiency is achieved. Yet, the buyer gets a rent when he knows that we are in the good state (which happens with probability $\nu \rho$ ). Observe that in scenario $C$ the buyer cannot be (strictly) better off than in scenario $U$.

Again, once we have found the optimal direct revelation mechanism that yields the seller's maximum expected profit, we can construct simple contracts that lead to the same outcome. Specifically, note that the seller can attain her maximum expected profit by offering to sell at a price $p$, unless the buyer proves that he obtained the signal $\sigma=L$. In the latter case, the price is reduced to $R_{L}$. The buyer can then either accept to buy at the price $p$, or provide evidence for the signal $\sigma=L$ and buy at the price $R_{L}$, or reject the offer. If $\nu>\hat{\nu}(\rho)$, it is optimal for the seller to set $p=R_{H}$. If $\nu \leq \hat{\nu}(\rho)$, it is optimal for her to set $p=E[R]$. 


\subsection{Certifiable vs. uncertifiable information}

We can now state our first main result, which follows from comparing the two parties' total profits derived in Propositions 1 and 2.

Proposition 3 (i) If $\nu \leq \hat{\nu}(\rho)$ and $\nu \geq \bar{\nu}(\rho)$, then the expected total surplus in scenario $U$ is the same as in scenario $C$.

(ii) If $\nu>\hat{\nu}(\rho)$ and $\nu \leq \tilde{\nu}(\rho)$, then the expected total surplus is smaller in scenario $C$ than in scenario $U$.

(iii) Otherwise, the expected total surplus is smaller in scenario $U$ than in scenario C.

The result is illustrated in Figure 1. Observe that if the probability $\rho$ that we are in the good state of the world is relatively small (the green area), trade always takes place in both scenarios, so the expected total profits are $S_{U}=S_{C}=E[R]$. Intuitively, when it is sufficiently likely that we are in the bad state of the world, the seller does not want to risk losing opportunities to trade by demanding a larger payment. Hence, ex post efficiency is achieved.

Now suppose that the probability $\nu$ that the buyer has private information is relatively small, but the good state has a sufficiently large probability $\rho$ (the blue area). In the case of uncertifiable information, the seller excludes the buyer of type $R_{L}$, because by doing so the seller can demand a payment $E[R]$ instead of only $R_{L}$. Yet, in the case of certifiable information, the seller can demand the payment $E[R]$ and reduce it to $R_{L}$ whenever the buyer provides evidence for $\sigma=L$, so trade always takes place. Hence, $S_{U}<S_{C}=E[R]$.

Next, suppose that the buyer is informed with a large probability and the good state is very likely (the brown area). When information is uncertifiable, trade occurs only if the buyer knows that we are in the good state. The seller asks for the largest possible payment $R_{H}$, since it is rather unlikely that the buyer will reject. When information is certifiable, the seller can offer to reduce the payment to $R_{L}$ whenever the buyer provides evidence for $\sigma=L$, so only an uninformed buyer is excluded from trade. As a result, $S_{U}<S_{C}<E[R]$. 
Finally, let us consider the most interesting case. Suppose that the buyer is informed with a large probability, but the probability of the good state is small (the yellow area). In the case of uncertifiable information, trade always takes place, because the seller does not want to risk losing opportunities to trade by demanding a payment larger than $R_{L}$. Yet, in the case of certifiable information, the seller can demand the larger payment $R_{H}$ and offer to reduce it to $R_{L}$ whenever the buyer provides evidence for $\sigma=L$. The seller thus excludes an uninformed buyer, but it is profitable to do so since the probability that the buyer is uninformed is relatively small. Therefore, $S_{C}<S_{U}=E[R]$, so certifiable information can lead to a strictly smaller total profit than uncertifiable information.

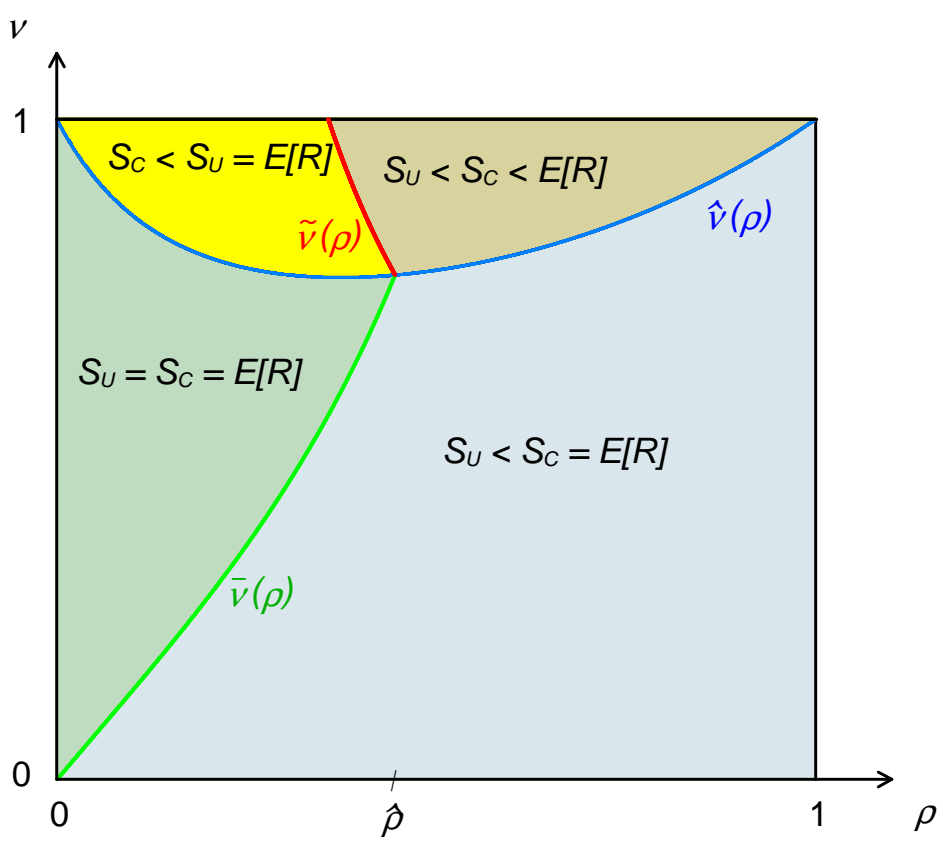

Figure 1. Exogenous information. Comparison of the expected total surplus in scenario $U$ (uncertifiable information) and scenario $C$ (certifiable information), depending on $\rho$ (the probability of the good state of the world) and $\nu$ (the probability that the buyer is informed). 


\section{Endogenous information}

So far, we have assumed that at date 0 the buyer learns the state of the world $\omega$ with some exogenously given probability $\nu$. At first glance, this assumption might appear unfamiliar, because in many adverse selection models with uncertifiable information it is assumed that the agent knows his type with certainty. ${ }^{13}$ We now show that an information structure where the buyer is informed with a probability strictly between 0 and 1 may indeed arise endogenously when the buyer can gather information before the contract is offered. Specifically, suppose that at date 0 the buyer decides whether or not to incur information acquisition costs $c>0$ in order to learn the state of the world. The buyer's decision is a hidden action, i.e. it cannot be observed by the seller (regardless of whether we are in scenario $U$ or in scenario C). ${ }^{14}$ If the buyer incurs the information acquisition costs, he learns the true state of the world $(\sigma=\omega)$, while otherwise he remains uninformed $(\sigma=\emptyset)$. It will turn out that in equilibrium the buyer may well acquire information with a probability $\nu \in(0,1)$.

\subsection{Scenario $U$ : Uncertifiable information}

Consider the case of uncertifiable information, where the buyer is free to make any claim $\hat{\sigma} \in\{H, L, \emptyset\}$ about the signal $\sigma$. First observe that in equilibrium the buyer will not always acquire information. To see this, suppose to the contrary the seller believes that $\nu=1$, so that $\nu>\hat{\nu}(\rho)$. According to Proposition 1(i), if $1>\tilde{\nu}(\rho)$ the seller would offer a contract that completely extracts the buyer's expected date- 2 profit. Thus, the buyer's profit at date 0 would be $0-c$. The buyer would prefer to deviate by remaining uninformed, so that his expected profit would be 0 . According to Proposition 1 (iii), if $1 \leq \tilde{\nu}(\rho)$ the buyer's expected profit at date 0

\footnotetext{
${ }^{13}$ Of course, in settings with certifiable information there would be no interesting screening problem if the agent knew his type with certainty. The principal could then simply insist on revelation of the information as a prerequisite for trade.

${ }^{14}$ We thus follow Crémer and Khalil (1992) and most of the subsequent literature on screening models with endogenous information. See Kessler (1998) and Hoppe (2013) for models in which a principal can observe an agent's decision to gather information. Note that this literature does not consider certifiable information.
} 
is $E[R]-R_{L}-c$ when he acquires information, while it would be $E[R]-R_{L}$ when he deviated. Hence, in any case the buyer would prefer to remain uninformed, so in equilibrium $\nu<1$ must hold.

To simplify the notation, we make the following definition.

Definition 2 Let $\psi(\rho)=\left(R_{H}-E[R]\right) \rho$.

Observe that there are parameter constellations such that in equilibrium the buyer never acquires information. Suppose the seller believes that $\nu=0$. In this case, given the seller's optimal contract offer according to Proposition 1(ii), the buyer's expected date- 0 profit is 0 , while it would be $\left(R_{H}-E[R]\right) \rho-c$ if the buyer deviated by acquiring information. Hence, if $c \geq \psi(\rho)$ it is indeed optimal for the buyer not to acquire information.

Now suppose that $c<\psi(\rho)$, so that in equilibrium the buyer must acquire information with a probability strictly between zero and one; i.e., he must be indifferent between acquiring and not acquiring information. Since the seller will always set $q_{H}=1$, the buyer's expected profit when he acquires information is $\rho\left(R_{H}-t_{H}\right)+(1-\rho)\left(q_{L} R_{L}-t_{L}\right)-c$, while it is $q_{\emptyset} E[R]-t_{\emptyset}$ if he does not acquire information. Recall that the transfer payments are given by (1), (2), and (3). Hence, the buyer is indifferent between acquiring and not acquiring information when $q_{\emptyset}-q_{L}=\frac{c}{\psi(\rho)}$, which lies strictly between zero and one. Inspection of Lemma 1 reveals that this condition can be satisfied only if $\nu=\hat{\nu}(\rho)$ or $\nu=\bar{\nu}(\rho)$. Hence, the following result must hold. ${ }^{15}$

Proposition 4 Consider scenario U (uncertifiable information).

(i) If $c \geq \psi(\rho)$, the buyer remains uninformed $(\nu=0)$. The seller offers the menu $\left(q_{H}=1, t_{H}=E[R]\right),\left(q_{L}=0, t_{L}=0\right),\left(q_{\emptyset}=1, t_{\emptyset}=E[R]\right)$. The seller's expected profit is $E[R]$, the buyer's expected profit is 0 , and hence the expected total surplus is $S_{U}=E[R]$.

(ii) If $c<\psi(\rho)$ and $\rho \leq \hat{\rho}$, the buyer acquires information with probability $\nu=$ $\bar{\nu}(\rho)$. The seller offers $\left(q_{H}=1, t_{H}=\frac{c}{\psi(\rho)} E[R]+\left(1-\frac{c}{\psi(\rho)}\right) R_{L}\right),\left(q_{L}=1-\frac{c}{\psi(\rho)}\right.$,

\footnotetext{
${ }^{15}$ Note that in the knife-edge case $c<\psi(\rho)$ and $\rho=\hat{\rho}$, due to Lemma 1(iv) the seller is indifferent between various equilibria. We assume that in this case the most efficient equilibrium is selected.
} 
$\left.t_{L}=\left(1-\frac{c}{\psi(\rho)}\right) R_{L}\right),\left(q_{\emptyset}=1, t_{\emptyset}=\frac{c}{\psi(\rho)} E[R]+\left(1-\frac{c}{\psi(\rho)}\right) R_{L}\right)$. The seller's expected profit is $R_{L}$, the buyer's expected profit is $E[R]-R_{L}-\frac{c}{1-\rho}$, and the expected total surplus is $S_{U}=E[R]-\frac{c}{1-\rho}$.

(iii) If $c<\psi(\rho)$ and $\rho>\hat{\rho}$, the buyer acquires information with probability $\nu=$ $\hat{\nu}(\rho)$. The seller offers $\left(q_{H}=1, t_{H}=\frac{c}{\psi(\rho)} E[R]+\left(1-\frac{c}{\psi(\rho)}\right) R_{H}\right),\left(q_{L}=0, t_{L}=0\right)$, $\left(q_{\emptyset}=\frac{c}{\psi(\rho)}, t_{\emptyset}=\frac{c}{\psi(\rho)} E[R]\right)$. The seller's expected profit is $\hat{\nu}(\rho) \rho R_{H}$, the buyer's expected profit is 0 , and the expected total surplus is $S_{U}=\hat{\nu}(\rho) \rho R_{H}$.

Now that we have derived the seller's maximum expected profits, we can again provide simple contracts that achieve the optimum. It is intuitively clear that when the information acquisition costs are prohibitively large (case i), the buyer will remain uninformed. In this case, a posted-price mechanism with $p=E[R]$ is optimal. When the information acquisition costs are sufficiently small, the equilibrium is in mixed strategies. Specifically, if the probability of the good state of the world is small (case ii), the buyer acquires information with probability $\bar{\nu}(\rho)$, and the seller mixes between the posted prices $p=E[R]$ (which is offered with probability $\left.\frac{c}{\psi(\rho)}\right)$ and $p=R_{L}$ (which is offered otherwise). Note that the buyer will always accept the offer, except when he learned the signal $\sigma=L$ and the price $p=E[R]$ is offered. Hence, the same expected payoffs will be attained as with the direct revelation mechanism characterized in part (ii) of the proposition. If the probability of the good state is large (case iii), the buyer acquires information with probability $\hat{\nu}(\rho)$, and the seller mixes between the posted prices $p=E[R]$ (which is offered with probability $\left.\frac{c}{\psi(\rho)}\right)$ and $p=R_{H}$ (which is offered otherwise). It is again easy to verify that in this way the same expected payoffs will be attained as with the direct revelation mechanism derived in part (iii) of the proposition.

\subsection{Scenario $C$ : Certifiable information}

Now consider the case of certifiable information, where the buyer cannot claim to have observed $\sigma=L$ or $\sigma=H$ without presenting the underlying evidence, while the buyer can always claim to be uninformed $(\sigma=\emptyset)$. It is again easy to see that in equilibrium the buyer will not always acquire information. Indeed, 
suppose the seller believed that $\nu=1$. According to Proposition 2(i), the seller would offer a contract that extracts the buyer's expected date-2 profit. Hence, the buyer's expected profit at date 0 would be $0-c$. The buyer would prefer to deviate by remaining uninformed, so that his expected profit would be 0 . Thus, in equilibrium $\nu<1$ must hold.

Yet, there are parameter constellations such that the buyer never acquires information. Suppose the seller believes that $\nu=0$. According to Proposition 2(ii), the seller offers a contract such that the buyer's expected profit is 0. When $c \geq \psi(\rho)$, deviating by acquiring information is not a profitable strategy for the buyer, since then his expected profit at date 0 would be $\psi(\rho)-c \leq 0$.

It follows that if $c<\psi(\rho)$, then in equilibrium the buyer must acquire information with a probability $\nu$ strictly between zero and one. Recall that the seller will always set $q_{L}=q_{H}=1$, so the buyer is indifferent between acquiring and not acquiring information whenever $\rho\left(R_{H}-t_{H}\right)+(1-\rho)\left(R_{L}-t_{L}\right)-c=q_{\emptyset} E[R]-t_{\emptyset}$ holds. Using (4), (5), and (6), this condition can be rewritten as $q_{\emptyset}=\frac{c}{\psi(\rho)}$. Since $0<\frac{c}{\psi(\rho)}<1$, Lemma 2 implies that in equilibrium $\nu=\hat{\nu}(\rho)$ must hold. Hence, we have derived the following result.

Proposition 5 Consider scenario $C$ (certifiable information).

(i) If $c \geq \psi(\rho)$, the buyer remains uninformed $(\nu=0)$. The seller offers the menu $\left(q_{H}=1, t_{H}=E[R]\right),\left(q_{L}=1, t_{L}=R_{L}\right),\left(q_{\emptyset}=1, t_{\emptyset}=E[R]\right)$. The seller's expected profit is $E[R]$, the buyer's expected profit is 0 , and hence the expected total surplus is $S_{C}=E[R]$.

(ii) If $c<\psi(\rho)$, the buyer acquires information with probability $\nu=\hat{\nu}(\rho)$. The seller offers $\left(q_{H}=1, t_{H}=\frac{c}{\psi(\rho)} E[R]+\left(1-\frac{c}{\psi(\rho)}\right) R_{H}\right),\left(q_{L}=1, t_{L}=R_{L}\right),\left(q_{\emptyset}=\frac{c}{\psi(\rho)}\right.$, $\left.t_{\emptyset}=\frac{c}{\psi(\rho)} E[R]\right)$. The seller's expected profit is $\hat{\nu}(\rho) E[R]$, the buyer's expected profit is 0 , and the expected total surplus is $S_{C}=\hat{\nu}(\rho) E[R]$.

Intuitively, when the information acquisition costs are sufficiently large, the buyer will not gather information. A simple contract that yields the same expected payoffs as the direct revelation mechanism derived in case (i) is a posted-price contract with $p=E[R]$, where the price is reduced to $R_{L}$ if and only if the buyer 
provides evidence that he obtained the signal $\sigma=L$. If the information acquisition costs are large (case ii), the buyer gathers information with probability $\nu=\hat{\nu}(\rho)$, and the seller mixes between the aforementioned contract (which is offered with probability $\left.\frac{c}{\psi(\rho)}\right)$ and a similar contract with $p=R_{H}$ (which is offered otherwise).

\subsection{Certifiable vs. uncertifiable information}

We can now compare the expected total profits derived in Propositions 4 and 5. Observe that there exists a threshold function

$$
\hat{c}(\rho):=\frac{(1-\rho) E[R]}{E[R]+\psi(\rho)} \psi(\rho),
$$

such that $\hat{\nu}(\rho) E[R]$ is smaller (larger) than $E[R]-\frac{c}{1-\rho}$ whenever $c$ is smaller (larger) than $\hat{c}(\rho)$.

Proposition 6 (i) If $c \geq \psi(\rho)$, or if $\rho \leq \hat{\rho}$ and $c=\hat{c}(\rho)$, then the expected total surplus in scenario $U$ is the same as in scenario $C$.

(ii) If $\rho \leq \hat{\rho}$ and $c<\hat{c}(\rho)$, then the expected total surplus is smaller in scenario $C$ than in scenario $U$.

(iii) Otherwise, the expected total surplus is smaller in scenario $U$ than in scenario C.

The result is illustrated in Figure 2. If the information acquisition costs are prohibitively large, then in both scenarios the buyer will refrain from strategically acquiring information (the blue area). In this case, the buyer is always willing to pay $E[R]$ and ex post efficiency is achieved, so $S_{U}=S_{C}=E[R]$. Now suppose that the costs of acquiring information are sufficiently small. It is not surprising that there are parameter constellations (the green area and the brown area) such that the expected total profit is smaller in the case of uncertifiable information, $S_{U}<S_{C}<E[R]$. 


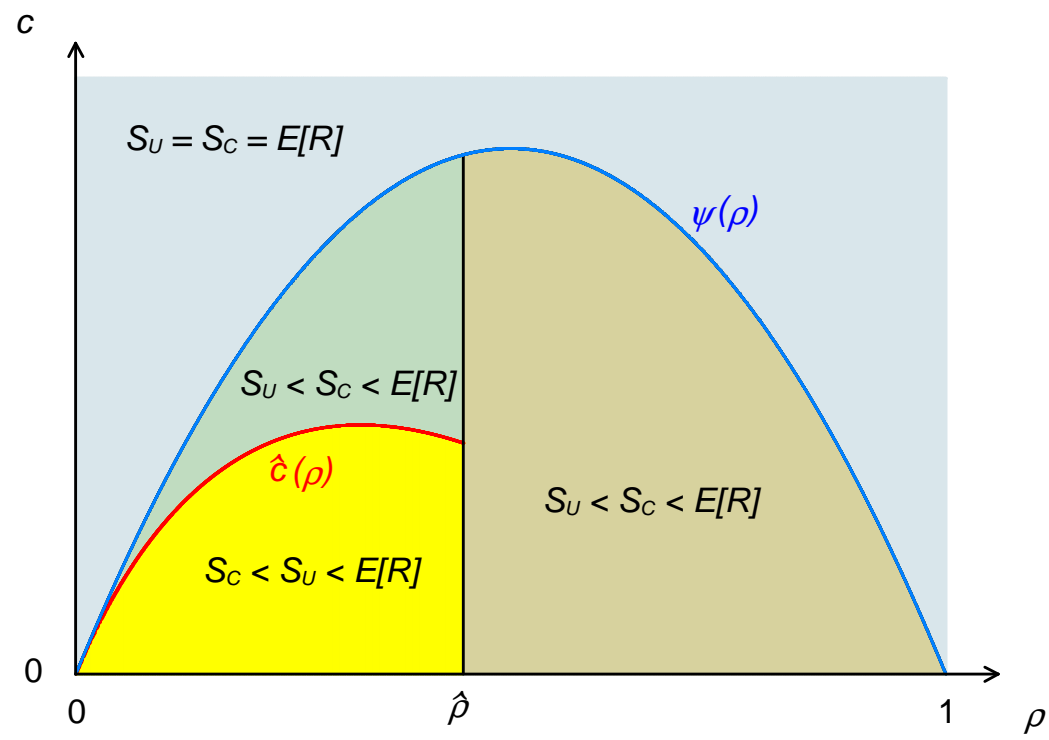

Figure 2. Endogenous information. Comparison of the expected total surplus in scenario $U$ (uncertifiable information) and scenario $C$ (certifiable information), depending on $\rho$ (the probability of the good state of the world) and $c$ (the costs of acquiring information).

Yet, Proposition 6(ii) shows that when the probability of the good state is small, certifiable information can lead to an even larger efficiency loss than uncertifiable information (the yellow area). This is the most interesting case. Specifically, trade always takes place when the buyer knows that we are in the good state. When information is uncertifiable, the seller mixes between the prices $E[R]$ and $R_{L}$, so an uninformed buyer is never excluded. In contrast, when information is certifiable, the seller mixes between the prices $E[R]$ and $R_{H}$, since she can offer to reduce the price to $R_{L}$ whenever the buyer provides evidence for the signal $\sigma=L$. As a consequence, an uninformed buyer may be excluded, which explains why $S_{C}<S_{U}<E[R]$ can hold.

Observe that the yellow area in Figure 2 (where the information structure is endogenous) corresponds qualitatively to the yellow area in Figure 1 (where the information structure is exogenous). In both cases, the expected total surplus given certifiable information can be smaller than the expected total surplus given 
uncertifiable information when the probability of the good state is relatively small and when the buyer is informed with a relatively large probability (which happens when the information gathering costs are small in the case of an endogenous information structure).

\section{Discussion}

In reality, the nature of private information held by firms may well be different from the nature of private information that a consumer has about his or her taste. Hence, screening models with uncertifiable information may not always capture the specifics of private information in contracting between firms. For example, in an M\&A context the management of a target company might have verifiable evidence about the value of its intangible assets. In such cases, the buyer may thus have certifiable information. Moreover, the buyer may decide whether or not to acquire such information for strategic reasons. The present paper has shown that even though the buyer has fewer possibilities to make false claims in the case of certifiable information, the resulting frictions may sometimes be even more severe than those caused by uncertifiable information.

We hope that the present paper will encourage new follow-up research, employing both theoretical and experimental methods.

First, from a standard theory perspective, one might study more general signal structures and information acquisition technologies. ${ }^{16}$ Moreover, while we have focused on opportunistic information acquisition, future research may also study productive information gathering. Furthermore, while we have considered takeit-or-leave-it contract offers, the implications of more general bargaining games could be explored.

Second, the behavioral literature has argued that decision makers often have

\footnotetext{
${ }^{16}$ For example, another way to model information acquisition by the buyer would be to let him discover the true state of the world only with a probability $\pi<1$. Note that if $\pi \leq \hat{\nu}(\rho)$, then in the case of certifiable information according to Proposition 2(ii) the buyer gets an information rent even when it is known that he always gathers information. Thus, in contrast to the situation studied in Proposition 5, for sufficiently small information acquisition costs the buyer's expected profit can then be strictly positive also in the case of certifiable information.
} 
a preference for honesty. While screening problems would become irrelevant if everyone were completely honest, a more realistic view might be that some people have a bad conscience when misrepresenting information, but find it less repugnant to conceal information. ${ }^{17}$ Hence, even when information is uncertifiable, people might behave as if we were in the case of certifiable information. Note that our results imply that the expected total surplus given such partially honest decision makers can be even smaller than the expected total surplus given completely opportunistic decision makers.

Third, experimental research might explore the relevance of this behavioral interpretation. Moreover, Hoppe and Schmitz (2013b) seems to be the only laboratory experiment so far that investigates a screening problem with endogenous information gathering. Thus, there is ample scope for experimental work exploring the differences between certifiable and uncertifiable information in situations where information can be endogenously acquired.

Fourth, private information is especially likely to be relevant when assets are of an innovative nature. Thus, it could be worthwhile to study the different implications of certifiable and uncertifiable information for the incentives to make innovations. $^{18}$

Fifth, in some instances disclosure laws might influence whether a buyer acquires certifiable or uncertifiable information. Future research exploring the interplay between the institutional framework and the nature of acquired information seems to be very promising. ${ }^{19}$

\footnotetext{
${ }^{17}$ Such preferences would be in the spirit of Spranca et al. (1991), who report that people often rate harmful omissions as less immoral than harmful commissions.

${ }^{18}$ For instance, there is a recent debate about whether public-private partnerships can foster innovations (see e.g. Carbonara and Pellegrino, 2020, and the literature discussed there). A prominent example of a public-private partnership is President Trump's "Operation Warp Speed", which has enabled the United States to rapidly develop and distribute vaccines against the novel coronavirus that recently originated in China. While the role of uncertifiable information for incentives to innovate in public-private partnerships has been studied in the literature (cf. Hoppe and Schmitz, 2013a; Buso, 2019), I am not aware of any papers that explore certifiable information in this context.

${ }^{19}$ In this context it might also be worthwhile to study screening problems with hybrid information structures where verification is costly, as in the literature on debt contracts initiated by Townsend (1979). On disclosure laws, see also the recent work by Schweizer (2017) and the literature discussed there.
} 
Finally, in the property rights approach to the theory of the firm (Grossman and Hart, 1986; Hart and Moore, 1990; Hart, 1995) it is usually assumed that there is symmetric information, so ex post efficiency is always achieved. In contrast, Williamson $(2000,2002)$ has emphasized the importance of ex post inefficiencies in transaction cost economics. Some authors have studied the role of asymmetric information in hold-up problems and in variants of the GrossmanHart-Moore approach, which can explain ex post inefficiencies (see e.g. Schmitz, 2006; Goltsman, 2011; Goldlücke and Schmitz, 2014; Su, 2017b). ${ }^{20}$ Yet, these papers consider uncertifiable information only. In future work, we plan to explore the role of certifiable information in the property rights approach to the theory of the firm.

\footnotetext{
${ }^{20}$ For alternative approaches to ex post inefficiencies, see also Mori (2017) on haggling and Mori (2020) on ex post adaptations.
} 


\section{Appendix}

Observation 1. The solution to the seller's relaxed problem in Section 4.1 [where only the constraints $\left(I C_{H \emptyset}\right),\left(I C_{\emptyset L}\right),\left(P C_{L}\right)$, and $q_{L} \leq q_{\emptyset} \leq q_{H}$ were considered] is also the solution to the seller's original problem, because it satisfies the omitted constraints $\left(I C_{H L}\right),\left(I C_{L H}\right),\left(I C_{L \emptyset}\right),\left(I C_{\emptyset H}\right),\left(P C_{H}\right)$, and $\left(P C_{\emptyset}\right)$.

Proof. If we plug in the expressions for the transfer payments $t_{L}, t_{\emptyset}$, and $t_{H}$ that were derived in (1), (2), and (3), all omitted constraints turn out to be satisfied: ${ }^{21}$

$$
\begin{gathered}
q_{H} R_{H}-t_{H} \geq q_{L} R_{H}-t_{L} \\
\Leftrightarrow q_{\emptyset}\left(R_{H}-E[R]\right)+q_{L}\left(E[R]-R_{L}\right) \geq q_{L} R_{H}-q_{L} R_{L} \\
\Leftrightarrow q_{\emptyset}\left(R_{H}-E[R]\right) \geq q_{L}\left(R_{H}-E[R]\right) \\
q_{L} R_{L}-t_{L} \geq q_{H} R_{L}-t_{H} \\
\Leftrightarrow 0 \geq q_{H} R_{L}-q_{H} R_{H}+q_{\emptyset}\left(R_{H}-E[R]\right)+q_{L}\left(E[R]-R_{L}\right) \\
\Leftrightarrow 0 \geq q_{H} R_{L}-q_{H} R_{H}+q_{\emptyset}\left(R_{H}-\left(\rho R_{H}+(1-\rho) R_{L}\right)\right) \\
+q_{L}\left(\left(\rho R_{H}+(1-\rho) R_{L}\right)-R_{L}\right) \\
\Leftrightarrow 0 \geq\left(R_{L}-R_{H}\right)\left(q_{H}-q_{\emptyset}+\left(q_{\emptyset}-q_{L}\right) \rho\right) \\
\Leftrightarrow q_{L}\left(E[R]-R_{L}\right) \geq q_{H} E[R]-q_{H} R_{H}+q_{\emptyset}\left(R_{H}-E[R]\right)+q_{L}\left(E[R]-R_{L}\right) \\
\Leftrightarrow 0\left(q_{H}-q_{\emptyset}\right)\left(E[R]-R_{H}\right) \\
q_{\emptyset} E[R]-t_{\emptyset} \geq q_{H} E[R]-t_{H}-t_{L} \geq q_{\emptyset} R_{L}-t_{\emptyset} \\
\Leftrightarrow q_{\emptyset} R_{L}-q_{\emptyset} E[R]+q_{L}\left(E[R]-R_{L}\right) \\
\Leftrightarrow 0\left(q_{\emptyset}-q_{L}\right)\left(R_{L}-E[R]\right)
\end{gathered}
$$

\footnotetext{
${ }^{21}$ To see this, recall that in the solution $q_{L} \leq q_{\emptyset} \leq q_{H}$ holds.
} 


$$
\begin{gathered}
q_{H} R_{H}-t_{H} \geq 0 \\
\Leftrightarrow q_{\emptyset}\left(R_{H}-E[R]\right)+q_{L}\left(E[R]-R_{L}\right) \geq 0 \\
q_{\emptyset} E[R]-t_{\emptyset} \geq 0 \\
\Leftrightarrow q_{L}\left(E[R]-R_{L}\right) \geq 0
\end{gathered}
$$

Observation 2. There exists a critical value $\hat{\rho} \in(0,1)$, such that $\rho \leq \hat{\rho}$ implies $\bar{\nu}(\rho) \leq \hat{\nu}(\rho) \leq \tilde{\nu}(\rho)$, and $\rho \geq \hat{\rho}$ implies $\tilde{\nu}(\rho) \leq \hat{\nu}(\rho) \leq \bar{\nu}(\rho)$.

Proof. First note that

$$
\begin{gathered}
\bar{\nu}(\rho) \leq \hat{\nu}(\rho) \\
\Leftrightarrow \frac{E[R]-R_{L}}{E[R](1-\rho)} \leq \frac{E[R]}{E[R]+\left(R_{H}-E[R]\right) \rho} \\
\Leftrightarrow E[R](1-\rho) E[R]-\left(E[R]+\left(R_{H}-E[R]\right) \rho\right)\left(E[R]-R_{L}\right) \geq 0 \\
\Leftrightarrow(1-\rho) R_{L} E[R]-\rho\left(E[R]-R_{L}\right) R_{H} \geq 0 .
\end{gathered}
$$

Next, observe that

$$
\begin{gathered}
\hat{\nu}(\rho) \leq \tilde{\nu}(\rho) \\
\Leftrightarrow \frac{E[R]}{E[R]+\left(R_{H}-E[R]\right) \rho} \leq \frac{R_{L}}{\rho R_{H}} \\
\Leftrightarrow\left(E[R]+\left(R_{H}-E[R]\right) \rho\right) R_{L}-E[R] \rho R_{H} \geq 0 \\
\Leftrightarrow(1-\rho) R_{L} E[R]-\rho\left(E[R]-R_{L}\right) R_{H} \geq 0 .
\end{gathered}
$$

Hence, it holds that $\bar{\nu}(\rho) \leq \hat{\nu}(\rho)$ whenever $\hat{\nu}(\rho) \leq \tilde{\nu}(\rho)$. Now define

$$
f(\rho):=(1-\rho) R_{L} E[R]-\rho\left(E[R]-R_{L}\right) R_{H} .
$$

Recall that $E[R]=\rho R_{H}+(1-\rho) R_{L}$ by definition. Hence, $f(\rho)=\left(R_{L}^{2}-R_{H}^{2}\right) \rho^{2}+$ $\left(R_{H} R_{L}-2 R_{L}^{2}\right) \rho+R_{L}^{2}$. A straightforward intermediate value argument implies 
that there must exist a unique $\hat{\rho} \in(0,1)$ such that $f(\hat{\rho})=0$, because $f(0)=$ $R_{L}^{2}>0, f(1)=\left(R_{L}-R_{H}\right) R_{H}<0$, and $f^{\prime \prime}(\rho)=2\left(R_{L}^{2}-R_{H}^{2}\right)<0$. 


\section{References}

Baron, D.P., Myerson, R.B., 1982. Regulating a monopolist with unknown costs. Econometrica 50, 911-930.

Bolton, P., Dewatripont, M., 2005. Contract Theory. MIT Press.

Buso, M., 2019. Bundling versus unbundling: Asymmetric information on information externalities. Journal of Economics 128, 1-25.

Carbonara, N., Pellegrino, R., 2020. The role of public private partnerships in fostering innovation. Construction Management and Economics 38, 140156.

Crémer, J., Khalil, F., 1992. Gathering information before signing a contract. American Economic Review 82, 566-578.

Crémer, J., Khalil, F., Rochet, J.-C., 1998a. Strategic information gathering before a contract is offered. Journal of Economic Theory 81, 163-200.

Crémer, J., Khalil, F., Rochet, J.-C., 1998b. Contracts and productive information gathering. Games and Economic Behavior 25, 174-193.

Goldlücke, S., Schmitz, P.W., 2014. Investments as signals of outside options. Journal of Economic Theory 150, 683-708.

Goltsman, M., 2011. Optimal information transmission in a holdup problem. Rand Journal of Economics 42, 495-526.

Green, J.R., Laffont, J.-J., 1986. Partially verifiable information and mechanism design. Review of Economic Studies 53, 447-456.

Grossman, S.J., Hart, O.D., 1980. Disclosure laws and takeover bids. Journal of Finance 35, 323-334.

Grossman, S.J., Hart, O.D., 1986. The costs and benefits of ownership: A theory of vertical and lateral integration. Journal of Political Economy 94, 691-719.

Hart, O.D., 1995. Firms, Contracts and Financial Structure. Oxford: Oxford University Press.

Hart, O., Holmström, B., 1987. The theory of contracts. In: Bewley, T. (ed.), Advances in Economics and Econometrics, Econometric Society Monographs, Fifth World Congress. Cambridge: Cambridge University Press, 71-155. 
Hart, O.D., Moore, J., 1990. Property rights and the nature of the firm. Journal of Political Economy 98, 1119-1158.

Hoppe, E.I., 2013. Observability of information acquisition in agency models. Economics Letters 119, 104-107.

Hoppe, E.I. and Schmitz, P.W., 2010. The costs and benefits of additional information in agency models with endogenous information structures. Economics Letters 107, 58-62.

Hoppe, E.I. and Schmitz, P.W., 2013a, Public-private partnerships versus traditional procurement: Innovation incentives and information gathering, Rand Journal of Economics 44, 56-74.

Hoppe, E.I., Schmitz, P.W., 2013b. Contracting under incomplete information and social preferences: An experimental study. Review of Economic Studies $80,1516-1544$.

Iossa, E., Martimort, D., 2015. Pessimistic information gathering. Games and Economic Behavior 91, 75-96.

Kessler, A.S., 1998. The value of ignorance. Rand Journal of Economics 29, 339-354.

Laffont, J.-J., Martimort, D., 1999. Separation of regulators against collusive behavior. Rand Journal of Economics 30, 232-262.

Laffont, J.-J., Martimort, D., 2002. The Theory of Incentives. Princeton University Press.

Lewis, T.R., Sappington, D.E., 1993. Ignorance in agency problems. Journal of Economic Theory 61, 169-183.

Lewis, T.R., Sappington, D.E., 1997. Information management in incentive problems. Journal of Political Economy 105, 796-821.

Maskin, E., Riley, J., 1984. Monopoly with incomplete information. Rand Journal of Economics 15, 171-196.

Milgrom, P.R., 1981. Good news and bad news: Representation theorems and applications. Bell Journal of Economics 12, 380-391.

Mori, Y., 2017. A formal model of firm boundaries and haggling. Economics Letters 156, 15-17. 
Mori, Y., 2020. Ex ante investment, ex post adaptation, and joint ownership. Economics Letters 187, 108927.

Myerson, R.B., 1982. Optimal coordination mechanisms in generalized principalagent problems. Journal of Mathematical Economics 10, 67-81.

Okuno-Fujiwara, M., Postlewaite, A., Suzumura, K., 1990. Strategic information revelation. Review of Economic Studies 57, 25-47.

Schmitz, P.W., 2006. Information gathering, transaction costs, and the property rights approach. American Economic Review 96, 422-434.

Schmitz, P.W., 2007. Optimal selling strategies when buyers may have hard information. European Economic Review 51, 859-870.

Schweizer, U., 2017. Incentives to acquire information under mandatory versus voluntary disclosure. Journal of Law, Economics, and Organization 33, 173-192.

Spranca, M., Minsk, E., Baron, J., 1991. Omission and commission in judgment and choice. Journal of Experimental Social Psychology 27, 76-105.

Su, A.P.-J., 2017a. Information acquisition and the equilibrium incentive problem. Journal of Economics and Management Strategy 26, 231-256.

$\mathrm{Su}$, A.P.-J., 2017b. Information revelation in the property right theory of the firms. International Journal of Industrial Organization 52, 133-164.

Tirole, J., 1986. Hierarchies and bureaucracies: On the role of collusion in organizations. Journal of Law, Economics, and Organization 2, 181-214.

Townsend, R.M., 1979. Optimal contracts and competitive markets with costly state verification. Journal of Economic Theory 21, 265-293.

Williamson, O.E., 2000. The new institutional economics: Taking stock, looking ahead. Journal of Economic Literature 38, 595-613.

Williamson, O.E., 2002. The theory of the firm as governance structure: From choice to contract. Journal of Economic Perspectives 16, 171-195.

Ye, B. and Li, S., 2018. Competitive contracts with productive information gathering. Journal of Economics 124, 1-17. 